\title{
Preparation of Scanning Tunneling Microscopy Tips Using Pulsed Alternating Current Etching
}

\author{
Victor A. Valencia \\ Avesh A. Thaker \\ Johnathan Derouin \\ Damian N. Valencia \\ Rachael G. Farber
}

See next page for additional authors

Follow this and additional works at: https://ecommons.luc.edu/chemistry_facpubs

Part of the Chemistry Commons

\section{Recommended Citation}

Valencia, Victor A.; Thaker, Avesh A.; Derouin, Johnathan; Valencia, Damian N.; Farber, Rachael G.; Gebel, Dana A.; and Killelea, Daniel. Preparation of Scanning Tunneling Microscopy Tips Using Pulsed Alternating Current Etching. Journal of Vacuum Science and Technology A, 33, 2: 1-4, 2015. Retrieved from Loyola eCommons, Chemistry: Faculty Publications and Other Works, http://dx.doi.org/10.1116/1.4904347

This Article is brought to you for free and open access by the Faculty Publications and Other Works by Department at Loyola eCommons. It has been accepted for inclusion in Chemistry: Faculty Publications and Other Works by an authorized administrator of Loyola eCommons. For more information, please contact ecommons@luc.edu. cc) (i) $\Theta$

This work is licensed under a Creative Commons Attribution-Noncommercial-No Derivative Works 3.0 License. (c) American Vacuum Society, 2015. 


\section{Authors}

Victor A. Valencia, Avesh A. Thaker, Johnathan Derouin, Damian N. Valencia, Rachael G. Farber, Dana A. Gebel, and Daniel Killelea 


\title{
BRIEF REPORTS AND COMMENTS
}

This section is intended for the publication of $(l)$ brief reports which do not require the formal structure of regular journal

articles, and (2) comments on items previously published in the journal.

\section{Preparation of scanning tunneling microscopy tips using pulsed alternating current etching}

\author{
Victor A. Valencia, Avesh A. Thaker, Jonathan Derouin, Damian N. Valencia, \\ Rachael G. Farber, Dana A. Gebel, and Daniel R. Killelea ${ }^{\text {a) }}$ \\ Department of Chemistry and Biochemistry, Loyola University Chicago, 1068 W. Sheridan Rd., \\ Chicago, Illinois 60660
}

(Received 19 September 2014; accepted 3 December 2014; published 18 December 2014)

\begin{abstract}
An electrochemical method using pulsed alternating current etching (PACE) to produce atomically sharp scanning tunneling microscopy (STM) tips is presented. An Arduino Uno microcontroller was used to control the number and duration of the alternating current (AC) pulses, allowing for ready optimization of the procedures for both Pt:Ir and W tips using a single apparatus. W tips prepared using constant and pulsed AC power were compared. Tips fashioned using PACE were sharper than those etched with continuous AC power alone. Pt:Ir tips were prepared with an initial coarse etching stage using continuous AC power followed by fine etching using PACE. The number and potential of the finishing AC pulses was varied and scanning electron microscope imaging was used to compare the results. Finally, tip quality using the optimized procedures was verified by UHV-STM imaging. With PACE, at least $70 \%$ of the W tips and $80 \%$ of the Pt:Ir tips were of sufficiently high quality to obtain atomically resolved images of HOPG or $\mathrm{Ni}(111)$. () 2014 American Vacuum Society. [http://dx.doi.org/10.1116/1.4904347]
\end{abstract}

\section{INTRODUCTION}

The scanning tunneling microscope (STM) has firmly established itself as a powerful technique for surface characterization and analysis. ${ }^{1-3}$ Central to the technique is the fact that the resolution of the instrument is dependent on the sharpness of the tip; in order to obtain images of surfaces with the component atoms clearly discerned, an atomically sharp tip is required. ${ }^{4}$ Reliable fabrication of quality tips has been an issue since the development of the STM, ${ }^{5}$ and over the past 30 years, many techniques have been developed to provide reliable and robust tip preparation methods. The techniques range widely, and often either require expensive, specialized equipment for implementation or rely on serendipity. For example, in the initial report of imaging with STM, the tips were formed by cutting a tungsten wire, then grinding the tip to a point, and finally crashing into the surface to "mini-spot weld" the tip to a fine point. ${ }^{5}$ Other approaches include simple cutting, ${ }^{6}$ ion-milling of the tips, ${ }^{7,8}$ and electrochemical etching. ${ }^{9-14}$ Of particular interest here is the electrochemical etching of tips; electrochemical etching of W and Pt:Ir wires is a promising method for the reliable, economical, fabrication of high purity and quality tips for STM. ${ }^{15-18}$

The objective was to use an electrochemical technique to fashion high quality tips with common, inexpensive equipment. Of particular note is the use of a commercially available, inexpensive, microcontroller with a USB connection to

${ }^{a}$ Electronic mail: dkillelea@luc.edu the computer and its packaged software. In comparison, other controlled tip etching techniques have employed DAQ computer boards and custom software, ${ }^{9}$ or other specialized equipment. ${ }^{19,20}$ The approach presented here is far less expensive and could be implemented in almost any facility. Electrochemical etching is attractive because it has been demonstrated to be effective in fashioning tips for many materials and applications. ${ }^{12,21}$ Furthermore, a high degree of tip purity can be realized by careful handling of the etching solution and equipment. Several etching techniques have been developed using both alternating current (AC) and direct current (DC) power sources, ${ }^{13,16}$ and while the details of the process for $\mathrm{AC}$ and $\mathrm{DC}$ etching differ, both are effective at fashioning STM tips. ${ }^{21,22}$

Electrochemical etching yields highly tapered tips with an extremely small radius of curvature $(<1 \mu \mathrm{m})$ because the etching rate of a wire dipped into an electrolytic solution is not uniform across the bottom face of the wire. ${ }^{18,19}$ When the tip wire was inserted into the solution, a meniscus forms, jutting up from the liquid surface. When an electric potential is applied to the tip wire, there is a concentration gradient of etchant ions within the meniscus that results in a vortex of the etchant ions about the tip apex as etching proceeds. The concentration of etchant ions is lowest at the top of the meniscus (body of the tip wire) and increases progressively toward the base (tapered tip); as a result, during the etching process, the etching rate is much faster at the base of the wire than along the sides. ${ }^{9}$ For DC etching, tip sharpness also depends on the ability to quickly shut off the power to 
the etching circuit at the moment the wire is etched to a point and drops off, because further etching dulls the tip. To achieve this aim, several techniques have been developed to quickly shut off the DC voltage at the drop-off point. ${ }^{6,17-19}$ Herein, an alternative approach is presented that utilizes pulses of $\mathrm{AC}$ to drive the etching process. A key advantage is that the eventual tip sharpness is not beholden to the ability to rapidly stop the etching process because the microcontroller program sends a set number of $\mathrm{AC}$ pulses. As a result, this technique reliably fashions atomically sharp tips for STM. We detail a simple apparatus that was used for the preparation of both $\mathrm{Pt}$ :Ir and $\mathrm{W}$ tips using the new, pulsed alternating current etching (PACE) technique.

\section{EXPERIMENT}

The PACE etching system used a variable AC power source (Variac) to set the AC potential, a graphite counter electrode ( $1 \mathrm{~cm}$ diameter graphite rod, McMaster-Carr), a custom made glass etching vessel (Allen Scientific Glass), and an Arduino Uno microcontroller (http://www.arduino.cc) that controlled the number and duration of AC pulses. The apparatus is depicted in Fig. 1(a). The glass-etching vessel [Fig. 1(b)] holds $\approx 100 \mathrm{ml}$ of etching solution, having a diameter of $70 \mathrm{~mm}$ and a depth of $30 \mathrm{~mm}$. The vessel design was similar to the apparatus presented by Hagedorn et al., ${ }^{15}$ and used a $15 \mathrm{~mm}$ diameter glass cylinder, with both the top and bottom open, fused to the inner wall of the vessel. The cylinder was $15 \mathrm{~mm}$ deep, and the vessel was filled so the liquid level was below the top of the small cylinder. The small glass cylinder contained any surface disturbances caused by bubbling at the graphite counter electrode. Therefore, etching occurred at a well-defined, fixed depth beneath etching solution, yielding high-quality tips. ${ }^{15}$

The Arduino Uno microcontroller was connected to a simple transistor and relay circuit, shown in Fig. 1(c). One of the digital $\mathrm{I} / \mathrm{O}$ pins $(\mathrm{I} / \mathrm{O}$ 13) on the Arduino Uno was used to

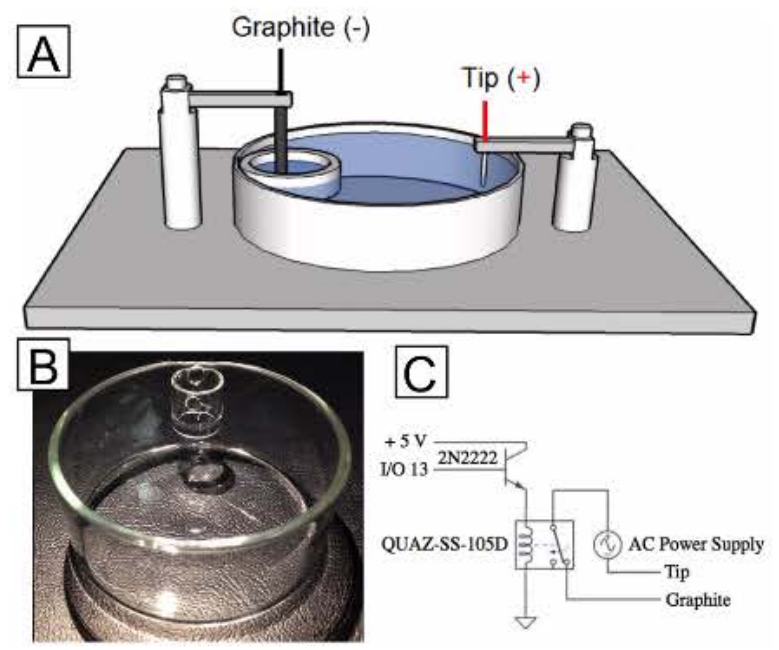

FIG. 1. (Color online) (a) Illustration of the electrochemical etching station showing the electrodes and arrangement of etching solution reservoirs; (b) photograph of the glass etching container; and (c) diagram of etching control circuit.

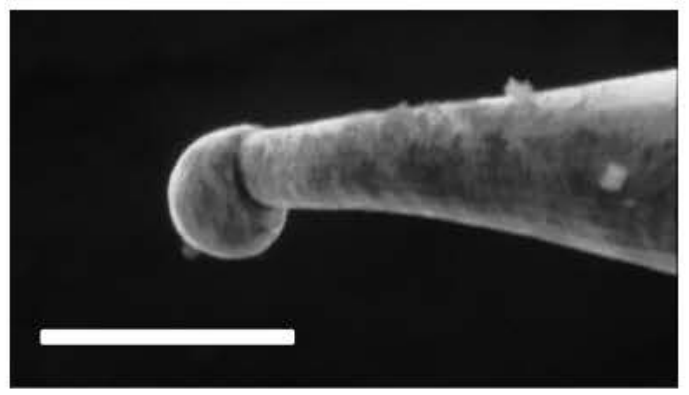

FIG. 2. SEM image of a Pt:Ir tip after coarse etching stage showing the bulbous tip remnant. Further fine etching removes the bulb and fashions a sharp tip. Scale bar is $20 \mu \mathrm{m}$.

activate a 2 N2222 transistor. The transistor, in turn, controlled a relay (Quaz-SS-105D) that switched the current flow from the Variac. The Arduino Uno microcontroller parameters were set using a packaged blinking LED control program (Arduino website), and allowed the relay to deliver AC voltage pulses of $0.5 \mathrm{~s}$ duration every $2 \mathrm{~s}$. The desired number of AC pulses was set in the software for tip fabrication once the optimal number of $\mathrm{AC}$ pulses was obtained from the optimization procedures detailed below. Pulse duration and frequency, as well as the potential and frequency $(60 \mathrm{~Hz})$ of the $\mathrm{AC}$ in the pulse, were measured during the etching process with a digital oscilloscope with $1 \mathrm{M} \Omega$ input impedance, and the RMS AC potential was verified with a handheld multimeter. We did not observe any undesired switching of the relay due to the etching current load or any extraneous pulses from noise in the circuit, and the pulse duration was largely invariant for all of the etches performed.

\section{RESULTS AND DISCUSSION}

\section{A. Platinum:Iridium Tips}

Pt:Ir tips were fashioned from $0.25 \mathrm{~mm}$ diameter $\mathrm{Pt}: \mathrm{Ir}$ wire $\left(80 \% \mathrm{Pt}, 20 \%\right.$ Ir) (Advent Materials) using $1.0 \mathrm{M} \mathrm{CaCl}_{2}$ (Aldrich, 99\%) as the etching solution. Etching of highquality Pt:Ir tips required two etching stages: an initial coarse stage using continuous AC power followed by a fine stage using PACE. The two-stage process was necessary because etching using PACE alone was time-consuming and therefore undesirable. Alternatively, etching using only continuous $\mathrm{AC}$ yielded tips with a bulbous deposit at the tip apex. A scanning electron microscope (SEM) image of the bulbous tip of a Pt:Ir wire etched using only continuous $\mathrm{AC}$ is shown in Fig. 2. When the two approaches were combined, quality tips were reliably fashioned. The coarse etching provided the gross shape, but left the bulbous mass. After coarse etching, the PACE stage was used to remove the bulb and prepared a tip suitable for STM imaging. A further benefit of using the two separate stages was that the overall etching time was appreciably reduced because a higher potential (faster etch rate) could be used for rough etching and the low-voltage, slower etching rate was only needed for the final fine sharpening.

The coarse etching stage was based on the Argonne Method $^{23}$ and consisted of three different voltage steps. The 


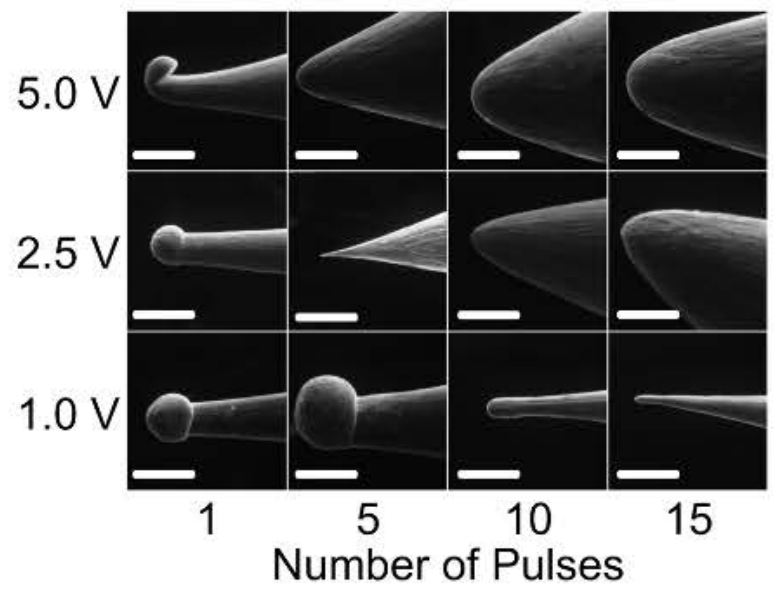

FIG. 3. SEM images of etched Pt:Ir tips after the fine etching (PACE) stage for several conditions of pulse voltage and number of pluses. The optimization of the technique is apparent; tips etching with a $1 \mathrm{~V}$ potential are not uniform, $5 \mathrm{~V}$ etching left a blunt tip, whereas at $2.5 \mathrm{~V}$, a sharp, uniform tip was observed after five pulses. White scale bars are $10 \mu \mathrm{m}$ in all images.

tip was first etched for $100 \mathrm{~s}$ using a continuous AC potential of $35 \mathrm{~V}$ (RMS). The potential was then dropped to $30 \mathrm{~V}$ (RMS) for $50 \mathrm{~s}$, and finally, the tips were etched at $28 \mathrm{~V}$ until the tip was almost entirely etched away and the bubbling ceased. Once coarse etching was completed, the tip was inserted 2-3 mm deeper into the solution and the fine etching step was performed. As shown in Fig. 3, after the same coarse etching was performed, the optimal fine etching PACE parameters were determined by varying the number and voltage of the pulses, and their effects on the tip shape were screened using SEM (Cambridge Instruments S240) imaging. Figure 3 shows a grid of representative SEM images illustrating how the optimal number of pulses and AC potential were determined. The optimal parameters were five pulses at $2.5 \mathrm{~V}$ (RMS) AC; these conditions consistently $(>80 \%)$ yielded sharp, symmetric tips. Etching above $2.5 \mathrm{~V}$, for example, $5.0 \mathrm{~V}$ (RMS), was able to rapidly remove the bulb formation from the coarse stage. However, the tips were persistently blunt, and did not achieve a similar degree of sharpness after 15 pulses (or for fewer than five pulses, data not shown), and further pulses had decreasing efficacy toward sharpening. In contrast, AC potentials below $2.5 \mathrm{~V}$ required more $\mathrm{AC}$ pulses to remove the bulbous remnants from the initial coarse stage, and once that was achieved, the tip quality rapidly degraded, becoming spindly and deformed, as shown in the SEM images for $1.0 \mathrm{~V}$ across the bottom of Fig. 3 .

\section{B. Tungsten Tips}

The PACE method was able to etch high-quality tungsten tips as well as Pt:Ir. The W tips were fashioned from $0.25 \mathrm{~mm}$ diameter $99.95 \%$ purity $\mathrm{W}$ wire (Advent Materials) using the setup shown in Fig. 1(a). $4.0 \mathrm{M} \mathrm{NaOH}$ (Aldrich, $99 \%$ ) solution (Nanopure water, $18 \mathrm{M} \Omega \mathrm{cm}$ ) was used as the etchant. As opposed to the two-step etching method used for Pt:Ir tips, the etching of high-quality W tips required only a single stage. The $\mathrm{W}$ wire was inserted to a depth of about
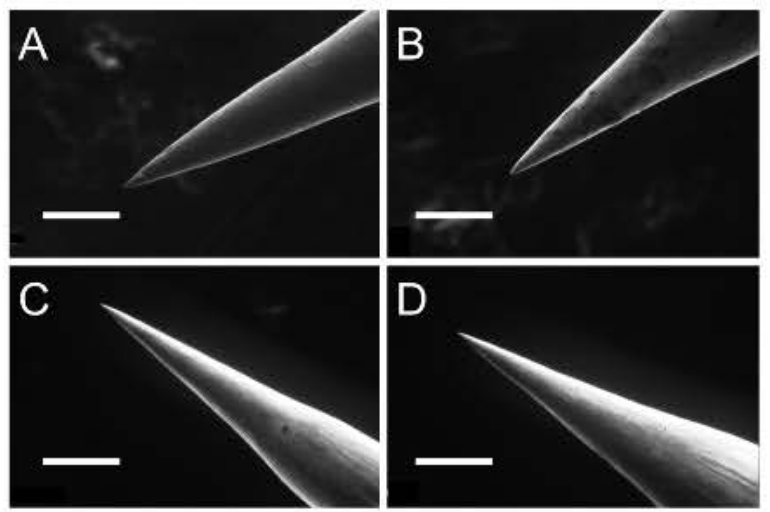

Fig. 4. SEM images of W tips etched with constant 5 VAC $[$ (a) and (b)] and pulsed 2.5 VAC [(c) and (d)]. The increased sharpness is evident in (c) and (d) with the pulsed AC etching technique. Scale bars are $20 \mu \mathrm{m}$ in all panels.

$3 \mathrm{~mm}$ below the liquid surface, with the meniscus forming around the wire. AC power was applied until bubbling from formation of $\mathrm{H}_{2}$ during the electrochemical etching of the tip ceased. $^{24}$ The tip was then extracted from the solution, rinsed with nanopure water and methanol, then dried in a stream of $\mathrm{N}_{2}$ gas. The etching solution could be used for up to ten tips with little loss in performance. However, after ten tips the etching time increased and the likelihood of impurities on the tip became more significant.

The abilities of the PACE method are highlighted by comparing the sharpness of $\mathrm{W}$ tips etched with continuous AC to those etched with the PACE method using SEM. Figure 4 shows images comparing tips etched with both approaches, using the same etching solution. With $\mathrm{W}$, the bulbous formation at the apex formed on Pt:Ir was not observed. Panels (a) and (b) in Fig. 4 show W tips etched with continuous AC power at a potential of $5 \mathrm{~V}$ (RMS); $5 \mathrm{~V}$ gave the highest quality tips for continuous AC. Typical etching times using continuous AC was around $90 \mathrm{~s}$. As seen in the figure, the tip geometry was symmetric, and a nice point was formed. In comparison, panels (c) and (d) of Fig. 4 show tips etched using PACE with a potential of $2.5 \mathrm{~V}$ (RMS) AC. From Fig. 4, it is apparent that the PACE tips were even sharper than those fashioned using continuous $\mathrm{AC}$, and they retained the highly symmetric shape indicative of a high-quality tip. For $\mathrm{W}$ tips, bulbous formations at the apex or other irregularities were seldom observed with AC etching.

With the SEM screening of the tip quality completed, the effectiveness of the PACE tip fabrication method was verified by obtaining atomically resolved images in a UHVSTM (RHK Technology, Pan Scan with R9 controller). Both $\mathrm{W}$ and Pt:Ir tips were prepared using PACE at a potential of $2.5 \mathrm{~V}$ (RMS) AC. The W tips were used to image an HOPG surface, and Pt:Ir tips were used to image both HOPG and Ni(111) surfaces; all imaging was done under UHV $\left(\mathrm{P}<1 \times 10^{-9}\right.$ Torr $)$ conditions. The Pt:Ir tips were used without any additional treatment, but $\mathrm{W}$ tips were heat treated prior to imaging. The STM chamber has a tip heating station where the tip is brought to within a few millimeters 

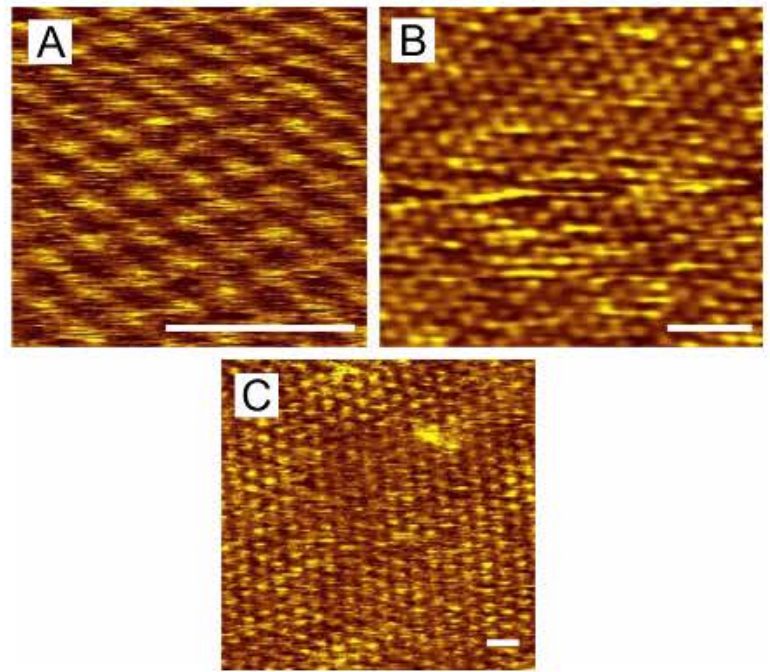

Fig. 5. (Color online) STM images of HOPG using (a) etched W tips $\left(2 \times 2 \mathrm{~nm}, T=80 \mathrm{~K}, V_{b}=55 \mathrm{mV}, I_{t}=400 \mathrm{pA}\right) ;($ b) Pt:Ir $(5 \times 5 \mathrm{~nm}, T=80 \mathrm{~K}$, $V_{b}=50 \mathrm{mV}, I_{t}=400 \mathrm{pA}$ ); (c) STM image of Ni(111) using etched Pt:Ir tip $\left(10 \times 10 \mathrm{~nm}, T=80 \mathrm{~K}, V_{b}=20 \mathrm{mV}, I_{t}=1.0 \mathrm{nA}\right)$. White scale bars are $1 \mathrm{~nm}$ for all images. The images are the unprocessed images obtained with the UHV-STM system.

of a white-hot $\mathrm{W}$ filament. The heating was used to remove any residual oxides on the $\mathrm{W}$ tips. ${ }^{25}$ As the tip was heated, the pressure rose from the base pressure of $7 \times 10^{-11}$ Torr into the low $10^{-9}$ Torr range, most likely a result of desorption from the tip holder. Heating was continued for approximately $1 \mathrm{~min}$ after the pressure in the chamber leveled off.

W tips etched using PACE were able to obtain atomically resolved images $70 \%$ of the time on $\mathrm{HOPG}$, out of a sample of ten tips. Figure 5(a) shows a representative, atomically resolved, STM image of HOPG where a treated W tip was used. The yield for Pt:Ir tips was even greater; at least $80 \%$ of the tips fabricated (out of 40 tips) using the pulsed AC technique gave atomic resolution on $\mathrm{HOPG}$, a representative STM image is shown in Fig. 5(b). Finally, the high quality of the Pt:Ir tips was further demonstrated by imaging a Ni(111) surface, as shown in Fig. 5(c). The high yield of at least $80 \%$ for quality Pt:Ir tips is in contrast to the typical $20 \%$ yield for Pt:Ir using the "cut-and-pull" technique in our lab (out of more than 100 tips). Tips etched using either continuous AC or suboptimal PACE parameters rarely yielded atomically resolved images, and therefore, exact statistics of their efficacy were not pursued.

\section{SUMMARY}

We have demonstrated that PACE is an inexpensive, straightforward electrochemical etching technique that reliably fashions tips for STM imaging. Both W and Pt:Ir tips were etched using the same apparatus, and at least $80 \%$ of the Pt:Ir tips $(70 \%$ of $\mathrm{W}$ tips) were capable of obtaining atomic resolution. Most notably, this system could be assembled with readily available equipment to etch either W and Pt:Ir tips by changing the etchant solution. The tip geometries after etching were screened with SEM imaging and the atomically resolved STM images demonstrated the high quality of the tips. PACE is clearly an economical, robust technique for the preparation of STM tips.

\section{ACKNOWLEDGMENTS}

This work was primarily supported by the College of Arts and Sciences at Loyola University Chicago. V. A. Valencia, J. Derouin, D. A. Gebel, and A. A. Thaker thank Loyola Undergraduate Research Opportunities (LUROP) for support through the Research Mentoring Program, Provost Fellowships, and Mulcahy Scholars Programs. Acknowledgment is made to the Donors of the American Chemical Society Petroleum Research Fund for partial support of this research through Grant PRF \#54770-DNI5.

'D. M. Eigler, P. S. Weiss, E. K. Schweizer, and N. D. Lang, Phys. Rev. Lett. 66, 1189 (1991).

${ }^{2}$ B. C. Stipe, M. A. Rezaei, and W. Ho, Science 280, 1732 (1998).

${ }^{3}$ J. Cerda, A. Michaelides, M. L. Bocquet, P. J. Feibelman, T. Mitsui, M. Rose, E. Fomin, and M. Salmeron, Phys. Rev. Lett. 93, 116101 (2004).

${ }^{4}$ J. Tersoff and D. R. Hamann, Phys. Rev. B 31, 805 (1985).

${ }^{5}$ G. Binnig, H. Rohrer, C. Gerber, and E. Weibel, Phys. Rev. Lett. 49, 57 (1982).

${ }^{6}$ A. J. Melmed, J. Vac. Sci. Technol., B 9, 601 (1991).

${ }^{7}$ M. J. Vasile, D. Grigg, J. E. Griffith, E. Fitzgerald, and P. E. Russell, J. Vac. Sci. Technol., B 9, 3569 (1991).

${ }^{8}$ S. W. Schmucker et al., Nat. Commun. 3, 935 (2012).

'R. Stone, M. Rosamond, K. Coleman, M. Petty, O. Kolosov, L. Bowen, V. Dubrovskii, and D. Zeze, Rev. Sci. Instrum. 84, 035107 (2013).

${ }^{10}$ D. Gingery and P. Buehlmann, Rev. Sci. Instrum. 78, 113703 (2007).

"'M. J. Zaccardi, K. Winkelmann, and J. A. Olson, J. Chem. Educ. 87, 308 (2010).

${ }^{12}$ B. Ren, G. Picardi, and B. Pettinger, Rev. Sci. Instrum. 75, 837 (2004).

${ }^{13}$ M. M. Jobbins, A. F. Raigoza, and S. A. Kandel, Rev. Sci. Instrum. 83, 036105 (2012).

${ }^{14}$ A. J. Nam, A. Teren, T. A. Lusby, and A. J. Melmed, J. Vac. Sci. Technol., B 13, 1556 (1995).

${ }^{15}$ T. Hagedorn, M. El Ouali, W. Paul, D. Oliver, Y. Miyahara, and P. Gruetter, Rev. Sci. Instrum. 82, 113903 (2011).

${ }^{16}$ B.-F. Ju, Y.-L. Chen, and Y. Ge, Rev. Sci. Instrum. 82, 013707 (2011).

${ }^{17}$ A. W. Liu, X. T. Hu, W. H. Liu, and G. J. Ji, Rev. Sci. Instrum. 68, 3811 (1997).

${ }^{18}$ J. P. Ibe, P. P. Bey, S. L. Brandow, R. A. Brizzolara, N. A. Burnham, D. P. Dilella, K. P. Lee, C. R. K. Marrian, and R. J. Colton, J. Vac. Sci. Technol., A 8, 3570 (1990).

${ }^{19}$ W.-T. Chang, I.-S. Hwang, M.-T. Chang, C.-Y. Lin, W.-H. Hsu, and J.-L. Hou, Rev. Sci. Instrum. 83, 083704 (2012).

${ }^{20}$ R. Fainchtein and P. R. Zarriello, Ultramicroscopy 42, 1533 (1992).

${ }^{21}$ G. J. Edwards and P. R. Pearce, J. Phys. D: Appl. Phys. 11, 761 (1978).

${ }^{22}$ S. L. Toh, H. Tan, J. C. Lam, L. C. Hsia, and Z. H. Mai, J. Electrochem. Soc. 157, E6 (2010).

${ }^{23}$ STM Tip Fabrication and Characterization, NST-SOP-122, Rev. 3, Argonne National Laboratory.

${ }^{24}$ G. Tahmasebipour, Y. Hoijat, V. Ahmadi, and A. Abdullah, Scanning 31, 65 (2009).

${ }^{25}$ Z. Q. Yu, C. M. Wang, Y. Du, S. Thevuthasan, and I. Lyubinetsky, Ultramicroscopy 108, 873 (2008). 